\title{
STUDENTS PERCEPTION TOWARDS THE USE OF VIRTUAL CLASSROOM OF RUMAH BELAJAR AND WHATSAPP FOR PROMOTING HIGHER ORDER THINKING SKILLS IN FLIPPED MATH CLASS
}

\author{
1) Ferdinand Wadu He, ${ }^{2}$ Arief Darmawan \\ 1) Mathematics Teacher, SMA Negeri 5 Kupang, Kupang City, East Nusa Tenggara \\ Province, Indonesia. \\ email : ferdinandwaduhe@gmail.com \\ 2) ICT Center for Education, Ministry of Education and \\ Culture, Indonesia. email:
}

arief.darmawan@kemdikbud.go.id

\begin{abstract}
The traditional classroom so-called lectures are still prevalent in most Indonesian classroom. Students of SMA Negeri 5 Kupang experience only 35 minutes' class periods because of morning and afternoon classroom shifts. This class period is too short and normally the focus is merely transferring content from the teachers to the students. Additionally, the traditional approach provides less opportunity for promoting students' Higher Order Thinking Skills (HOTS) which elaborated as critical in the K13 (Indonesian curriculum 2013). Flipped classroom approach was expected to help the teachers to deal with the aforementioned problem. In the current study, WhatsApp and Virtual Classroom of Rumah Belajar were used to flip the mathematics classroom. Thirty-eight focus group discussion with each group consists of 3-5 students 12 MIA 1-6 were employed in the data collection. Thematic analysis was used to analyse the qualitative data. The result showed that the majority of students perceived that both learning platforms were helpful to provide effectively efficient learning activities during the class period. Both learning management system allowed students to have an active learning that encouraged them to be more actively engaged during the 35 minutes' class period. The combined use of WhatsApp and Rumah Belajar shed the light to a greater opportunity for promoting students' Higher Order Thinking Skills.
\end{abstract}

Keywords: Flipped Classroom, Rumah Belajar, WhatsApp , Higher Order Thinking Skills (HOTS), Mathematics Classroom

\section{INTRODUCTION}

\section{RESEARCH CONTEXT}

This study was initiated from the problems occurred in learning and teaching mathematics at State Senior High School 5 Kupang City, East Nusa Tenggara Province. First of all, the challenge was students did not have a handbook for 12th grade mathematical specialization. The difficulty of getting student and teacher handbook for mathematical specialization encouraged researchers to arrive at an alternative solution for this problem which was downloading the K13 (Indonesian curriculum 2013) mathematical specialization e- book (softcopy) from the internet. The fact was the majority of students have gadgets such as smartphones and androids. Then, WhatsApp groups are made for the entire 12th grade MIA (Science Class) 1-6 in which the mathematical specialization e-book was then shared with all 
students. This was when the flipped classroom started with 12th grade MIA students whom then became the sample in this study.

Furthermore, the mathematics classroom was flipped using WhatsApp as a learning platform in six classes: Class 12 MIA 1 (36 students), 12 MIA 2 (32 students), 12 MIA 3 (34 students), 12 MIA 4 (36 students), 12 MIA 5 (31 students) and Class 12 MIA 6 (29 students) in State Senior High School 5 Kupang City, East Nusa Tenggara. WhatsApp media has been used for half-semester flipped Classroom, from June to August 2018. This platform seemed to be very helpful to facilitate learning activities because it allowed students to be more actively involved during learning with only 35-minute class period.

It cannot be denied that the sophistication of Information and Communication Technology (ICT) greatly influences the way the teacher conducts teaching and learning activities in the classroom. After 3 months running flipped classroom using WhatsApp, at the end of August 2018, the Rumah Belajar shed a new light for the flipped math classroom. The flipped classroom was applied using one feature at Rumah Belajar which was virtual classroom. The virtual classroom of Rumah Belajar was then employed to flip the math classroom settings. This learning management system was hoped to encourage students to be more active and more independent in their learning. Currently, a total of 198 participants of 12 MIA students actively use the virtual classroom of Rumah Belajar.

The flipped classroom unwittingly began to half-shifted from WhatsApp to the virtual classroom of Rumah Belajar. This triggered the researcher to investigate students' perception of the use of WhatsaApp and Rumah Belajar and student preferences as well as their reasons for both learning platforms which they perceive better between WhatsApp or the virtual classroom of Rumah Belajar in supporting the flipped maths classroom.

The second issue was the class period at SMA N 5 Kupang which was usually 45 minutes, now the allocation for learning time period in the classroom is only 35 minutes because teaching and learning activities are divided into two shifts, morning and afternoon classes. The sample in the current study itself is in the morning class. This period is very short and usually the focus is only on transferring learning content from teacher to student. Indeed, the limited time of face to face classroom setting provides less opportunities to promote students' Higher-Order Thinking Skills (HOTS). This problem cannot be left unsolved because the so-called HOTS is a highly demanded skill that needs to be mastered by Indonesian students. Because the skill has been described as something essential in K13 curriculum. The initial idea to introduce students to another class model,

from traditional classroom to flipped classroom, was expected to help the help the researcher dealing with the challenge in lacking of time efficiency in maths learning activities. More importantly, the use of flipped classroom was hoped to promote HOTS to $12 \mathrm{MIA}$ students at SMA N 5 Kupang.

This study is an ongoing study to first investigate students' perceptions about flipped classroom using WhatsApp and virtual classroom of Rumah Belajar. Secondly, this study also investigated students' preferences and their reasons for the learning management systems that they considered better between WhatsApp or virtual classroom of Rumah Belajar to support the math flipped classroom. Thirdly, it then explored students' perception towards the opportunities to promote students' High Order Thinking Skills (HOTS) by using the flipped classroom, either WhatsApp or Rumah Belajar.

\section{LITERATURE REVIEW}

Flipped classroom has three parts of the learning activity process, namely 1 ) pre classroom (online), 2) face to face classroom (face to face class) and 3) classroom (online) post. 
Flipped classroom is an innovative learning approach that changes the traditional way of teaching, allowing students to gain knowledge before face-to-face learning in the classroom. Learning in a flipped classroom allows learning material to be given outside the classroom (online) so that there will be much time for practice more exercises and learning engagement in the classroom (Strayer, 2012). This facilitates students to become more active and independent in the learning process, especially when working on questions that contain higher order thinking skills (HOTS) (Fulton, 2012). In fact, flipped classroom to facilitate students' learning can exist because of Information Communication and Technology (ICT) use (Amresh, Carberry, \& Femiani, 2013).

Livingstone in 2012 highlighted two fundamental problems about ICT in learning. The first was that the difficulties in distinguishing which aspects of ICT can promote learning for students. This is due to the various form of educational technologies under the umbrella of ICT. The second problem is the conventional perception of ICT where ICT is only used as a tool for drill and skill education which is equivalent to only promoting Lower Order Thinking Skills (LOTS) rather than HOTS (Livingstone, 2012). Therefore, further research is needed to investigate ICT as a learning media in promoting HOTS.

Regarding the use of ICT, digital media such as social media and instant messaging applications are rapid changing landscape of communication in this era. The existence of online media has a significant effect on how students learn and how teachers teach (Griesemer, 2014). However, regardless of the popular use of social media, only few educators use it to improve learning activities (Lenhart, Purcell, Smith, \& Zickuhr, 2010). Online

communication between groups of students and between students and teachers has become popular recently through several communication media such as: email, sms, facebook, twitter and WhatsApp (Liu, 2010). Using WhatsApp in flipped classroom could affect students' critical thinking skills (Sayan, 2016).

As Today's education tries to develop students' ability to think critically (HOTS), not only delivering course content (Kustijono \& Zuhri, 2018). The ICT offers teachers to transform the quality of teaching to be more student centered learning than teacher centered learning. By integrating ICT effectively, students with teachers collaborate in learning activities both in classroom and outside the classroom. In some previous studies, it was found that the use of ICT has a positive impact on students' learning outcomes and learning in small groups give more benefits for students for their learning processes (Falode et al., 2015; Martin \& Parker, 2014).

In Indonesian context, the Indonesian Ministry of Education and Culture through the Center for Information Communication and Technology called (Pustekkom) has launched an online learning platform namely Rumah Belajar that contains eight main educational features. One of them is Kelas Maya (virtual classroom) This feature allows Indonesian teachers to create and manage virtual classrooms as a free learning management system (LMS). Provided, it can be accessed for free, then it should not be wasted. This must be efficiently harnessed for the sake of student learning and teacher teaching. As the evidence, after thoroughly reviewing literatures, there was still limited educational research on the use of virtual classroom of Rumah Belajar to improve student learning outcomes, especially in promoting students' HOTS. 


\section{METHODOLOGY}

This research is a qualitative study with a total of 198 students of 12th grade MIA SMAN 5 Kupang, East Nusa Tenggara participated in this study. Details of the number of students per class as follows: Class 12 MIA 1 (36 students), 12 MIA 2 (32 students), 12 MIA 3 (34 students), 12 MIA 4 (36 students), 12 MIA 5 (31 students) and Class 12 MIA 6 (29 students).

The data collection was conducted through semi structured interview in focus group discussion consisting of all 12 MIA students of SMA N 5 Kupang City ( $n=198)$ who participated in flipped classroom activities. Focus group discussion is a data collection technique that relies on interviews with a small group of participants in collaborative and interactive sessions rather than individuals. Focus group discussion can be defined as "a small group of individuals who have the same interests or characteristics, mediated by moderators, who use groups and their interactions as a way to get information about a particular problem" (Williams \& Katz, 2001).

Thirty-Eight (38) focus group discussions with each group consisting of 3-5 students of 12 MIA 1-12 MIA 6 used in the data collection process. At the end of the Trigonometric Limit session, students were invited to participate in interactive sessions to gather feedback about their experiences in learning activities through WhatsApp and virtual classroom of Rumah Belajar including benefits, challenges and their preferences in using the two learning media platforms, WhatsApp and Virtual Classroom of Rumah Belajar. This session lasted around 30 minutes per focus group discussion.

Thematic analysis was used in analyzing data obtained through reflective feedback in the 38 focus group discussions above. At the end, the researchers also surveyed student preferences and their reasons for the media that they considered better whether WhatsApp or virtual classroom of Rumah Belajar in supporting the flipped maths classroom. 


\section{RESULT AND DISCUSSION}

After the data through semi structured interview from 38 focus group discussions was gathered, they were then analysed. The results then were described such as written in the table below.

Table 1. Students' perception and preference about the use of Whatsapp and Rumah Belajar in math flipped classroom.

\begin{tabular}{|c|c|c|c|c|c|}
\hline \multirow[t]{2}{*}{ FGD } & \multicolumn{2}{|c|}{ Whatsapp(WA) } & \multicolumn{2}{|c|}{ Rumah Belajar(RB) } & \multirow{2}{*}{$\begin{array}{l}\text { Pr } \\
\text { ef. }\end{array}$} \\
\hline & + & - & + & - & \\
\hline 1 & $\begin{array}{l}\text { 1) Quickly } \\
\text { accessed } \\
\text { 2) Time efficiency }\end{array}$ & $\begin{array}{l}\text { No } \\
\text { online } \\
\text { test }\end{array}$ & $\begin{array}{l}\text { 1) Students } \\
\text { became } \\
\text { more active } \\
\text { 2) Self paced } \\
\text { learning } \\
\text { 3) Self } \\
\text { reflection/ev } \\
\text { aluation } \\
\end{array}$ & $\begin{array}{l}\text { Technic } \\
\text { al issue } \\
\text { when } \\
\text { taking } \\
\text { the } \\
\text { online } \\
\text { test }\end{array}$ & $\begin{array}{l}W \\
A+ \\
R B\end{array}$ \\
\hline 2 & $\begin{array}{l}\text { 1) Communicative } \\
\text { 2) Quick response }\end{array}$ & $\begin{array}{l}\text { No } \\
\text { online } \\
\text { test }\end{array}$ & $\begin{array}{l}\text { 1) Students } \\
\text { activities } \\
\text { can be } \\
\text { monitore } \\
d \text { by the } \\
\text { teacher } \\
\text { 2) Exercise } \\
\text { for } \\
\text { practice } \\
\text { is } \\
\text { available }\end{array}$ & $\begin{array}{l}\text { Technic } \\
\text { al issue } \\
\text { when } \\
\text { taking } \\
\text { the } \\
\text { online } \\
\text { test }\end{array}$ & $\begin{array}{l}W \\
A+ \\
\text { RB }\end{array}$ \\
\hline 3 & $\begin{array}{l}\text { 1) Time efficiency } \\
\text { 2) Information } \\
\text { exchange }\end{array}$ & $\begin{array}{l}\text { No } \\
\text { online } \\
\text { test }\end{array}$ & $\begin{array}{l}\text { 1) Students } \\
\text { became } \\
\text { more active } \\
\text { 2) Time } \\
\text { efficiency } \\
\text { 3) National } \\
\text { exam } \\
\text { practice }\end{array}$ & $\begin{array}{l}\text { Technic } \\
\text { al issue } \\
\text { when } \\
\text { taking } \\
\text { the } \\
\text { online } \\
\text { test }\end{array}$ & $\begin{array}{l}W \\
A+ \\
\text { RB }\end{array}$ \\
\hline
\end{tabular}




\begin{tabular}{|c|c|c|c|c|c|}
\hline 4 & $\begin{array}{l}\text { 1) Students } \\
\text { became more } \\
\text { active } \\
\text { 2) Allows Q and A }\end{array}$ & $\begin{array}{l}\text { No } \\
\text { online } \\
\text { test }\end{array}$ & $\begin{array}{l}\text { 1) Online } \\
\text { national } \\
\text { exam } \\
\text { practice } \\
\text { 2) Time } \\
\text { efficiency }\end{array}$ & $\begin{array}{l}\text { Multiple } \\
\text { choice } \\
\text { questio } \\
\text { n }\end{array}$ & $\begin{array}{l}W \\
A+ \\
R B\end{array}$ \\
\hline 5 & $\begin{array}{l}\text { Quick response } \\
\text { by the teacher } \\
\text { when there is a } \\
\text { sudden } \\
\text { question }\end{array}$ & $\begin{array}{l}\text { No } \\
\text { online } \\
\text { test }\end{array}$ & $\begin{array}{l}\text { 1) Online } \\
\text { national } \\
\text { exam } \\
\text { practice } \\
\text { (containing } \\
\text { HOTS) } \\
\text { 2) Discussion } \\
\text { forum }\end{array}$ & $\begin{array}{l}\text { Technic } \\
\text { al issue } \\
\text { when } \\
\text { taking } \\
\text { the } \\
\text { online } \\
\text { test }\end{array}$ & $\mathrm{RB}$ \\
\hline 6 & $\begin{array}{l}\text { Quick response } \\
\text { by the teacher } \\
\text { when there is a } \\
\text { sudden } \\
\text { question }\end{array}$ & $\begin{array}{l}\text { No } \\
\text { online } \\
\text { test }\end{array}$ & $\begin{array}{l}\text { 1) Online } \\
\text { national } \\
\text { exam } \\
\text { practice } \\
\text { (containing } \\
\text { HOTS) } \\
\text { 2) Systematic } \\
\text { learning } \\
\text { activities } \\
\text { 3) Interesting } \\
\text { display }\end{array}$ & $\begin{array}{l}\text { Easily } \\
\text { get } \\
\text { distract } \\
\text { ed to } \\
\text { browse } \\
\text { unnece } \\
\text { ssary } \\
\text { things } \\
\text { on the } \\
\text { internet. }\end{array}$ & $\mathrm{RB}$ \\
\hline 7 & $\begin{array}{l}\text { There is a } \\
\text { notification on the } \\
\text { gadget so it is easy } \\
\text { to keep students } \\
\text { updated with new } \\
\text { information or } \\
\text { announcement. }\end{array}$ & $\begin{array}{l}\text { No } \\
\text { online } \\
\text { test }\end{array}$ & $\begin{array}{l}\text { 1) Online } \\
\text { national } \\
\text { exam } \\
\text { practice } \\
\text { (containing } \\
\text { HOTS) } \\
\text { 2) Number of } \\
\text { virtual } \\
\text { classes in } \\
\text { RB which } \\
\text { can be }\end{array}$ & $\begin{array}{l}\text { Easily } \\
\text { get } \\
\text { distract } \\
\text { ed to } \\
\text { browse } \\
\text { unnece } \\
\text { ssary } \\
\text { things } \\
\text { on the } \\
\text { internet. }\end{array}$ & $\mathrm{RB}$ \\
\hline
\end{tabular}




\begin{tabular}{|c|c|c|c|c|c|}
\hline & & & $\begin{array}{l}\text { attended by } \\
\text { students. }\end{array}$ & & \\
\hline 8 & $\begin{array}{l}\text { 1) Communicative } \\
\text { 2) simple }\end{array}$ & $\begin{array}{l}\text { No } \\
\text { online } \\
\text { test }\end{array}$ & $\begin{array}{l}\text { 1) Students } \\
\text { became } \\
\text { more } \\
\text { independent } \\
\text { 2) Students' } \\
\text { academic } \\
\text { performance } \\
\text { are getting } \\
\text { better than it } \\
\text { used to be. }\end{array}$ & $\begin{array}{l}\text { A bit } \\
\text { hard } \\
\text { when } \\
\text { teacher } \\
\text { s only } \\
\text { guide } \\
\text { student } \\
\text { in } \\
\text { online } \\
\text { discussi } \\
\text { on } \\
\text { forum }\end{array}$ & $\begin{array}{l}W \\
A+ \\
R B\end{array}$ \\
\hline 9 & Communicative & $\begin{array}{l}\text { No } \\
\text { online } \\
\text { test }\end{array}$ & $\begin{array}{l}\text { 1) Systematic } \\
\text { learning } \\
\text { activities } \\
\text { 2) Online } \\
\text { national } \\
\text { exam } \\
\text { practice } \\
\text { (containing } \\
\text { HOTS) } \\
\text { 3) Discussion } \\
\text { forum } \\
\text { 4) Students get } \\
\text { much time } \\
\text { to practice } \\
\text { in the } \\
\text { classroom. }\end{array}$ & $\begin{array}{l}\text { Technic } \\
\text { al issue } \\
\text { when } \\
\text { taking } \\
\text { the } \\
\text { online } \\
\text { test }\end{array}$ & RB \\
\hline 10 & Communicative & $\begin{array}{l}\text { No } \\
\text { online } \\
\text { test }\end{array}$ & $\begin{array}{l}\text { 1) Online } \\
\text { national } \\
\text { exam } \\
\text { practice } \\
\text { (containing } \\
\text { HOTS) }\end{array}$ & $\begin{array}{l}\text { Technic } \\
\text { al issue } \\
\text { when } \\
\text { taking } \\
\text { the }\end{array}$ & RB \\
\hline
\end{tabular}




\begin{tabular}{|c|c|c|c|c|c|}
\hline & & & $\begin{array}{l}\text { 2) Systematic } \\
\text { learning } \\
\text { activities } \\
\text { 3) Complete } \\
\text { learning } \\
\text { materials }\end{array}$ & $\begin{array}{l}\text { online } \\
\text { test }\end{array}$ & \\
\hline 11 & $\begin{array}{l}\text { Information } \\
\text { exchange }\end{array}$ & $\begin{array}{l}\text { Too } \\
\text { much } \\
\text { chats in } \\
\text { WA } \\
\text { group } \\
\text { can be } \\
\text { annoyin } \\
\text { g }\end{array}$ & $\begin{array}{l}\text { 1) Students } \\
\text { can access } \\
\text { other } \\
\text { features in } \\
\text { Rumah } \\
\text { Belajar } \\
\text { 2) Self paced } \\
\text { learning } \\
\text { 3) Students } \\
\text { are happy } \\
\text { because } \\
\text { they can } \\
\text { instantly } \\
\text { know their } \\
\text { marks on } \\
\text { online test }\end{array}$ & $\begin{array}{l}\text { Technic } \\
\text { al issue } \\
\text { when } \\
\text { taking } \\
\text { the } \\
\text { online } \\
\text { test }\end{array}$ & $\mathrm{RB}$ \\
\hline 12 & No need to login & $\begin{array}{l}\text { No } \\
\text { online } \\
\text { test }\end{array}$ & $\begin{array}{l}\text { 1) Students } \\
\text { experiences } \\
\text { e-Learning. } \\
\text { 2) Online } \\
\text { discussion } \\
\text { forum } \\
\text { 3) Students } \\
\text { are happy } \\
\text { because } \\
\text { they can } \\
\text { instantly }\end{array}$ & $\begin{array}{l}\text { Technic } \\
\text { al issue } \\
\text { when } \\
\text { taking } \\
\text { the } \\
\text { online } \\
\text { test }\end{array}$ & $\begin{array}{l}W \\
A+ \\
R B\end{array}$ \\
\hline
\end{tabular}

know their 


\begin{tabular}{|c|c|c|c|c|c|}
\hline & & & $\begin{array}{l}\text { marks on } \\
\text { online test } \\
\text { 4) Support the } \\
\text { face to face } \\
\text { classroom }\end{array}$ & & \\
\hline 13 & $\begin{array}{l}\text { 1) Time efficiency } \\
\text { 2) No need to } \\
\text { login }\end{array}$ & $\begin{array}{l}\text { Too } \\
\text { much } \\
\text { chats in } \\
\text { WA } \\
\text { group } \\
\text { makes } \\
\text { students } \\
\text { find it } \\
\text { difficult } \\
\text { to } \\
\text { search } \\
\text { for the } \\
\text { learning } \\
\text { material } \\
\text { that } \\
\text { have } \\
\text { been } \\
\text { posted } \\
\text { by the } \\
\text { teacher }\end{array}$ & $\begin{array}{l}\text { 1) Students } \\
\text { can access } \\
\text { other } \\
\text { features in } \\
\text { Rumah } \\
\text { Belajar } \\
\text { 2) Online } \\
\text { national } \\
\text { exam } \\
\text { practice } \\
\text { (containing } \\
\text { HOTS) } \\
\text { 3) Students get } \\
\text { used to } \\
\text { online exam } \\
\text { as } \\
\text { preparation } \\
\text { for facing } \\
\text { UNBK }\end{array}$ & $\begin{array}{l}\text { Technic } \\
\text { al issue } \\
\text { to login } \\
\text { into } \\
\text { Rumah } \\
\text { Belajar }\end{array}$ & $\mathrm{RB}$ \\
\hline 14 & $\begin{array}{l}\text { Information } \\
\text { exchange }\end{array}$ & $\begin{array}{l}\text { Less } \\
\text { systema } \\
\text { tic }\end{array}$ & $\begin{array}{l}\text { 1) More } \\
\text { effective } \\
\text { than WA } \\
\text { 2) More } \\
\text { systematic } \\
\text { than WA } \\
\text { 3) Online } \\
\text { national } \\
\text { exam } \\
\text { practice } \\
\text { (containing }\end{array}$ & $\begin{array}{l}\text { Technic } \\
\text { al } \\
\text { problem } \\
\text { in } \\
\text { userna } \\
\text { me and } \\
\text { passwo } \\
\text { rd entry }\end{array}$ & $\begin{array}{l}W \\
A+ \\
\text { RB }\end{array}$ \\
\hline
\end{tabular}




\begin{tabular}{|c|c|c|c|c|c|}
\hline 15 & Quick response & $\begin{array}{l}\text { No } \\
\text { online } \\
\text { test }\end{array}$ & $\begin{array}{l}\text { 1) Online } \\
\text { national } \\
\text { exam } \\
\text { practice } \\
\text { (containing } \\
\text { HOTS } \\
\text { 2) Offers much } \\
\text { time to } \\
\text { practice } \\
\text { more } \\
\text { questions } \\
\text { contain } \\
\text { HOTS } \\
\text { 3) Students } \\
\text { became } \\
\text { more } \\
\text { enthusiastic } \\
\text { for taking an } \\
\text { online test } \\
\text { because } \\
\text { they can } \\
\text { instantly } \\
\text { know the } \\
\text { result. }\end{array}$ & $\begin{array}{l}\text { Technic } \\
\text { al } \\
\text { problem } \\
\text { in } \\
\text { userna } \\
\text { me and } \\
\text { passwo } \\
\text { rd entry }\end{array}$ & $\begin{array}{l}W \\
A+ \\
R B\end{array}$ \\
\hline 16 & $\begin{array}{l}\text { Information } \\
\text { exchange }\end{array}$ & $\begin{array}{l}\text { No } \\
\text { online } \\
\text { test }\end{array}$ & $\begin{array}{l}\text { Self paced } \\
\text { learning }\end{array}$ & - & $\begin{array}{l}W \\
A+ \\
R B\end{array}$ \\
\hline 17 & Quick response & $\begin{array}{l}\text { No } \\
\text { online } \\
\text { test }\end{array}$ & $\begin{array}{l}\text { Students can } \\
\text { access other } \\
\text { features in } \\
\text { Rumah Belajar }\end{array}$ & $\begin{array}{l}\text { Easily } \\
\text { get } \\
\text { distract } \\
\text { ed to } \\
\text { browse } \\
\text { unnece } \\
\text { ssary } \\
\text { things } \\
\text { on the }\end{array}$ & $\begin{array}{l}W \\
A+ \\
R B\end{array}$ \\
\hline
\end{tabular}




\begin{tabular}{|c|c|c|c|c|c|}
\hline 18 & $\begin{array}{l}\text { Information } \\
\text { exchange }\end{array}$ & $\begin{array}{l}\text { Less } \\
\text { systema } \\
\text { yc }\end{array}$ & $\begin{array}{l}\text { 1) Time } \\
\text { efficiency } \\
\text { 2) Learning } \\
\text { became } \\
\text { more } \\
\text { enjoyable } \\
\text { 3) Online test } \\
\text { familiarise } \\
\text { student with } \\
\text { the UNBK }\end{array}$ & $\begin{array}{l}\text { No } \\
\text { instant } \\
\text { notificat } \\
\text { ion on } \\
\text { student } \\
\text { s } \\
\text { gadget }\end{array}$ & $\begin{array}{l}W \\
A+ \\
R B\end{array}$ \\
\hline 19 & Quick response & $\begin{array}{l}\text { No } \\
\text { online } \\
\text { test }\end{array}$ & $\begin{array}{l}\text { Students } \\
\text { became more } \\
\text { active and } \\
\text { independent } \\
\text { learners }\end{array}$ & $\begin{array}{l}\text { Easily } \\
\text { get } \\
\text { distract } \\
\text { ed to } \\
\text { browse } \\
\text { unnece } \\
\text { ssary } \\
\text { things } \\
\text { on the } \\
\text { internet. }\end{array}$ & $\begin{array}{l}W \\
A+ \\
\text { RB }\end{array}$ \\
\hline 20 & $\begin{array}{l}\text { Information } \\
\text { exchange }\end{array}$ & $\begin{array}{l}\text { several } \\
\text { docume } \\
\text { nts } \\
\text { sometim } \\
\text { es can } \\
\text { not be } \\
\text { opened }\end{array}$ & $\begin{array}{l}\text { Self paced } \\
\text { learning }\end{array}$ & - & $\begin{array}{l}W \\
A+ \\
R B\end{array}$ \\
\hline 21 & $\begin{array}{l}\text { Information } \\
\text { exchange }\end{array}$ & $\begin{array}{l}\text { No } \\
\text { online } \\
\text { test }\end{array}$ & $\begin{array}{l}\text { Self paced } \\
\text { learning }\end{array}$ & $\begin{array}{l}\text { Easily } \\
\text { get } \\
\text { distract } \\
\text { ed to } \\
\text { browse } \\
\text { unnece } \\
\text { ssary }\end{array}$ & $\begin{array}{l}W \\
A+ \\
\text { RB }\end{array}$ \\
\hline
\end{tabular}

things 


\begin{tabular}{|c|c|c|c|c|c|}
\hline & & & & $\begin{array}{l}\text { on the } \\
\text { internet. }\end{array}$ & \\
\hline 22 & Time efficiency & $\begin{array}{l}\text { No } \\
\text { online } \\
\text { test }\end{array}$ & $\begin{array}{l}\text { Offers much } \\
\text { time in class to } \\
\text { practice } \\
\text { exercise which } \\
\text { containing } \\
\text { HOTS }\end{array}$ & - & $\begin{array}{l}W \\
A+ \\
R B\end{array}$ \\
\hline 23 & $\begin{array}{l}\text { Information } \\
\text { exchange }\end{array}$ & $\begin{array}{l}\text { No } \\
\text { online } \\
\text { test }\end{array}$ & $\begin{array}{l}\text { Offers much } \\
\text { time in class to } \\
\text { practice } \\
\text { exercise which } \\
\text { containing } \\
\text { HOTS }\end{array}$ & $\begin{array}{l}\text { Technic } \\
\text { al } \\
\text { problem } \\
\text { in } \\
\text { userna } \\
\text { me and } \\
\text { passwo } \\
\text { rd entry }\end{array}$ & $\begin{array}{l}W \\
A+ \\
R B\end{array}$ \\
\hline 24 & Quick response & $\begin{array}{l}\text { No } \\
\text { online } \\
\text { test }\end{array}$ & $\begin{array}{l}\text { 1) Complete } \\
\text { learning } \\
\text { material } \\
\text { 2) Systematic } \\
\text { learning } \\
\text { activities } \\
\text { 3) Online } \\
\text { discussion } \\
\text { forum }\end{array}$ & $\begin{array}{l}\text { Technic } \\
\text { al } \\
\text { problem } \\
\text { in } \\
\text { userna } \\
\text { me and } \\
\text { passwo } \\
\text { rd entry }\end{array}$ & $\begin{array}{l}W \\
A+ \\
R B\end{array}$ \\
\hline 25 & Quick response & $\begin{array}{l}\text { No } \\
\text { online } \\
\text { test }\end{array}$ & $\begin{array}{l}\text { Online test } \\
\text { familiarise } \\
\text { student with } \\
\text { the UNBK }\end{array}$ & - & $\begin{array}{l}W \\
A+ \\
R B\end{array}$ \\
\hline 26 & Quick response & $\begin{array}{l}\text { No } \\
\text { online } \\
\text { test }\end{array}$ & $\begin{array}{l}\text { 1) Online test } \\
\text { familiarise } \\
\text { student with } \\
\text { the UNBK } \\
\text { 2) Students } \\
\text { became }\end{array}$ & $\begin{array}{l}\text { No } \\
\text { drawba } \\
\text { cks }\end{array}$ & $\begin{array}{l}W \\
A+ \\
R B\end{array}$ \\
\hline
\end{tabular}




\begin{tabular}{|c|c|c|c|c|c|}
\hline & & & $\begin{array}{l}\text { more } \\
\text { motivated }\end{array}$ & & \\
\hline 27 & Quick response & $\begin{array}{l}\text { No } \\
\text { online } \\
\text { test }\end{array}$ & $\begin{array}{l}\text { Affordable } \\
\text { because no } \\
\text { need to buy } \\
\text { textbooks }\end{array}$ & - & $\begin{array}{l}W \\
A\end{array}$ \\
\hline 28 & $\begin{array}{l}\text { Information } \\
\text { exchange }\end{array}$ & $\begin{array}{l}\text { Less } \\
\text { systema } \\
\text { tic }\end{array}$ & $\begin{array}{l}\text { 1) Students } \\
\text { became } \\
\text { more } \\
\text { independent } \\
\text { 2) Online test } \\
\text { familiarise } \\
\text { student with } \\
\text { the UNBK } \\
\end{array}$ & $\begin{array}{l}\text { Technic } \\
\text { al issue } \\
\text { when } \\
\text { taking } \\
\text { the } \\
\text { online } \\
\text { test }\end{array}$ & RB \\
\hline 29 & $\begin{array}{l}\text { Information } \\
\text { exchange }\end{array}$ & $\begin{array}{l}\text { No } \\
\text { online } \\
\text { test }\end{array}$ & $\begin{array}{l}\text { 1) Online test } \\
\text { familiarise } \\
\text { student with } \\
\text { the UNBK } \\
\text { 2) Students } \\
\text { became } \\
\text { more active } \\
\text { and } \\
\text { independent } \\
\text { learners } \\
\text { 3) Students } \\
\text { are } \\
\text { equipped to } \\
\text { be more } \\
\text { critical } \\
\text { thinkers }\end{array}$ & $\begin{array}{l}\text { Easily } \\
\text { get } \\
\text { distract } \\
\text { ed to } \\
\text { browse } \\
\text { unnece } \\
\text { ssary } \\
\text { things } \\
\text { on the } \\
\text { internet. }\end{array}$ & $\begin{array}{l}W \\
A+ \\
R B\end{array}$ \\
\hline 30 & $\begin{array}{l}\text { Information } \\
\text { exchange }\end{array}$ & $\begin{array}{l}\text { No } \\
\text { online } \\
\text { test }\end{array}$ & $\begin{array}{l}\text { Offers much } \\
\text { time in class to } \\
\text { practice } \\
\text { exercise which } \\
\text { containing }\end{array}$ & - & RB \\
\hline
\end{tabular}

HOTS 


\begin{tabular}{|c|c|c|c|c|c|}
\hline $31 \mathrm{Ir}$ & $\begin{array}{l}\text { ormation } \\
\text { exchange and quick } \\
\text { response }\end{array}$ & - & $\begin{array}{l}\text { Offers much } \\
\text { time in class to } \\
\text { practice } \\
\text { exercise which } \\
\text { containing } \\
\text { HOTS }\end{array}$ & $\begin{array}{l}\text { No } \\
\text { drawba } \\
\text { cks }\end{array}$ & RB \\
\hline 32 & $\begin{array}{l}\text { Information } \\
\text { exchange and quick } \\
\text { response }\end{array}$ & $\begin{array}{l}\text { No } \\
\text { online } \\
\text { test }\end{array}$ & $\begin{array}{l}\text { Online national } \\
\text { exam practice } \\
\text { (containing } \\
\text { HOTS) }\end{array}$ & $\begin{array}{l}\text { Need to } \\
\text { regularl } \\
\text { y check } \\
\text { the RB } \\
\text { whether } \\
\text { there is } \\
\text { a new } \\
\text { announ } \\
\text { cement } \\
\text { or } \\
\text { informat } \\
\text { ion }\end{array}$ & $\begin{array}{l}\mathrm{W} \\
\mathrm{A}+ \\
\mathrm{RB}\end{array}$ \\
\hline 33 & $\begin{array}{l}\text { Information } \\
\text { exchange and quick } \\
\text { response }\end{array}$ & $\begin{array}{l}\text { No } \\
\text { online } \\
\text { test }\end{array}$ & $\begin{array}{l}\text { 1) Students } \\
\text { became } \\
\text { more } \\
\text { motivated in } \\
\text { learning } \\
\text { 2) Students } \\
\text { are able to } \\
\text { solve } \\
\text { questions } \\
\text { containing } \\
\text { HOTS }\end{array}$ & - & $\begin{array}{l}W \\
A+ \\
\text { RB }\end{array}$ \\
\hline 34 & Time efficiency & $\begin{array}{l}\text { No } \\
\text { online } \\
\text { test }\end{array}$ & $\begin{array}{l}\text { 1) Online } \\
\text { national } \\
\text { exam } \\
\text { practice }\end{array}$ & $\begin{array}{l}\text { Student } \\
\text { S } \\
\text { became } \\
\text { more } \\
\end{array}$ & $\begin{array}{l}W \\
A+ \\
R B\end{array}$ \\
\hline
\end{tabular}




\begin{tabular}{|c|c|c|c|c|c|}
\hline & & & $\begin{array}{l}\text { (containing } \\
\text { HOTS) } \\
\text { 2) Time } \\
\text { efficiency }\end{array}$ & $\begin{array}{l}\text { individu } \\
\text { al } \\
\text { learner } \\
\text { rather } \\
\text { than } \\
\text { social } \\
\text { learner } \\
\end{array}$ & \\
\hline 35 & quick response & $\begin{array}{l}\text { Internet } \\
\text { quota is } \\
\text { limited }\end{array}$ & $\begin{array}{l}\text { 1) Learning } \\
\text { became } \\
\text { more } \\
\text { interesting } \\
\text { 2) Self paced } \\
\text { learning }\end{array}$ & $\begin{array}{l}\text { Technic } \\
\text { al issue } \\
\text { when } \\
\text { taking } \\
\text { the } \\
\text { online } \\
\text { test }\end{array}$ & $\begin{array}{l}W \\
A+ \\
\text { RB }\end{array}$ \\
\hline 36 & practice & $\begin{array}{l}\text { No } \\
\text { online } \\
\text { test }\end{array}$ & $\begin{array}{l}\text { 1) Learning } \\
\text { materials } \\
\text { such as } \\
\text { videos are } \\
\text { helpful } \\
\text { 2) Learning } \\
\text { materials } \\
\text { are made } \\
\text { clear and } \\
\text { concise } \\
\end{array}$ & $\begin{array}{l}\text { Technic } \\
\text { al issue } \\
\text { when } \\
\text { taking } \\
\text { the } \\
\text { online } \\
\text { test }\end{array}$ & $\begin{array}{l}\mathrm{W} \\
\mathrm{A}+ \\
\mathrm{RB}\end{array}$ \\
\hline 37 & $\begin{array}{l}\text { Information } \\
\text { exchange and quick } \\
\text { response }\end{array}$ & $\begin{array}{l}\text { No } \\
\text { online } \\
\text { test }\end{array}$ & $\begin{array}{l}\text { 1) Learning } \\
\text { materials } \\
\text { are made } \\
\text { clear and } \\
\text { concise } \\
\text { 2) Offers much } \\
\text { time in class } \\
\text { to practice } \\
\text { exercise } \\
\text { which } \\
\text { containing }\end{array}$ & $\begin{array}{l}\text { Technic } \\
\text { al } \\
\text { problem } \\
\text { in } \\
\text { userna } \\
\text { me and } \\
\text { passwo } \\
\text { rd entry }\end{array}$ & $\begin{array}{l}W \\
A+ \\
\text { RB }\end{array}$ \\
\hline
\end{tabular}

HOTS 


\begin{tabular}{|c|c|c|c|c|c|}
\hline & & & $\begin{array}{l}\text { 3) Students } \\
\text { became } \\
\text { more } \\
\text { independent } \\
\text { learner } \\
\end{array}$ & & \\
\hline 38 & Time efficiency & $\begin{array}{l}\text { No } \\
\text { online } \\
\text { test }\end{array}$ & $\begin{array}{l}\text { Learning } \\
\text { materials } \\
\text { are made } \\
\text { clear and } \\
\text { concise }\end{array}$ & $\begin{array}{l}\text { Error } \\
\text { internet } \\
\text { connect } \\
\text { ion }\end{array}$ & $\begin{array}{l}W \\
A+ \\
R B\end{array}$ \\
\hline
\end{tabular}

$$
\begin{array}{ll}
\text { WA } & =\text { WhatsApp } \\
\text { RB } & \\
\text { FG } & =\text { Rumah Belajar } \\
& =\text { Focus Group Discussion }
\end{array}
$$

Based on the table 1 above, it can be seen that focus groups which favour the use of virtual classroom of Rumah Belajar because they perceived that Rumah Belajar are practical, systematic, well organized, offers online exam, allows students to immediately know their marks after taking the online exam, and provides online discussion forums that allow interactive communication between teacher-student and student-student.

This is explicitly stated from their statement in the focus group discussion as follows:

FGD 31: "We were able to access and grasp the learning material before face to face learning activities. Thus, in face to face classroom, the explanation given in the classroom is not rambling on or straight to the point."

FGD 32: "Learning material in virtual classroom of Rumah Belajar is systematically organized and is complete with details in learning activities 1, 2 and 3." 
FGD 33: "The virtual classroom of Rumah Belajar is educative. Rumah Belajar has a variety of features that make us more motivated to learn. The exercise is provided and it helps us to practice more. Since using Rumah Belajar, we have much time to learn independently and actively so that we can begin working on difficult questions that require us to manipulate information or use various formula to be able to solve it. This is of course with the assistance of our teacher"

Based on table 1, there are a total of 38 focus group discussions employed in this research. Meanwhile, the results on student preferences towards flipped math classroom using WhatsApp and Rumah Belajar can be shown in the Venn diagram below.

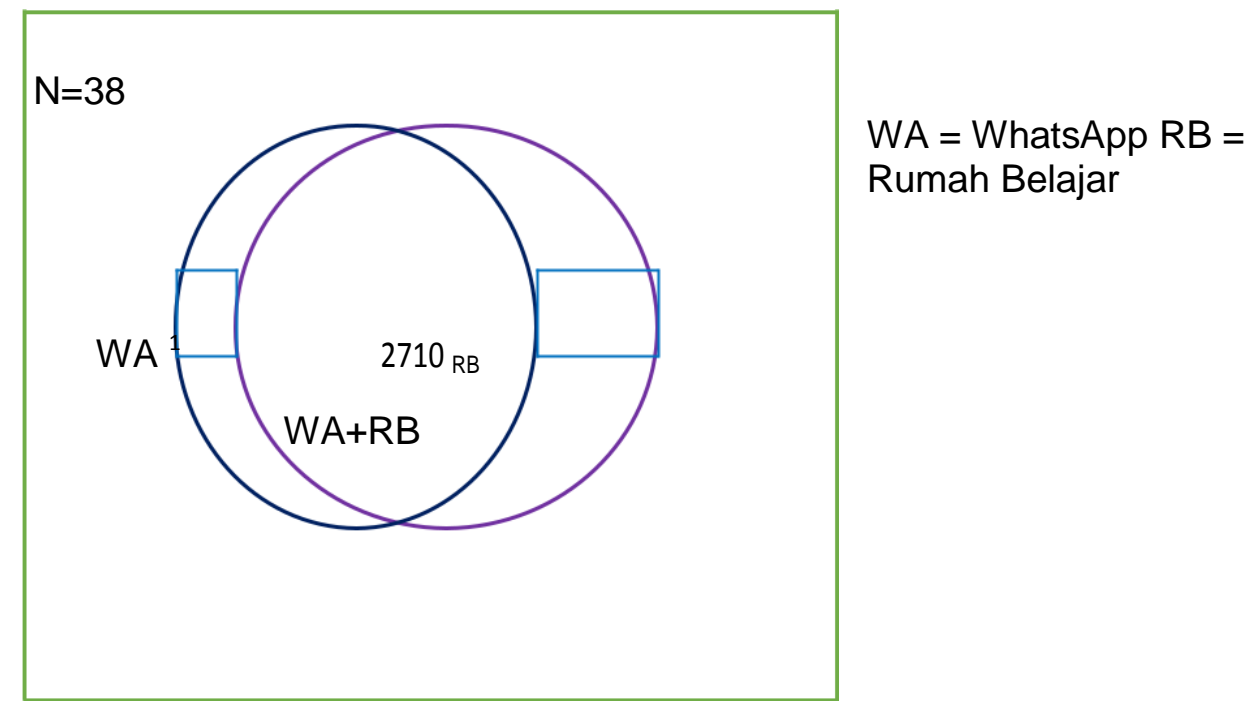

Figure 1. Students preferences towards WhatsApp and Rumah Belajar as flipped classroom platform

Based on the diagram above, it appears that there was only one group of a total of 38 focus group discussions who preferred using only WhatsApp without Rumah Belajar in their flipped classroom. 
Next, there are 10 groups who thought that they should only use Rumah Belajar without WhatsApp in their flipped math classroom.

It is obvious in the Venn diagram above that the majority of students with more than $50 \%$ of focus group discussion participants which is 27 groups preferred to use these two online learning platforms, WhatsApp and virtual class of Rumah Belajar simultaneously as needed in flipped classroom.

In general, 38 focus group discussions gave positive responses to the use of flipped classroom in their mathematics learning. Majority of students mentioned that getting the material first to be studied at home before face-to-face classes is efficient. They became better prepared to experience teaching and learning activities in the classroom. Rumah Belajar allowed them to be well engaged in the classroom and grasp any learning material discussed in class easily.

The majority of the focus groups mentioned that they were helped by the existence of virtual classroom of Rumah belajar especially in terms of the time efficiency for learning. The limited time period, 35 minutes available became more efficiently spent in face-to-face classes which used for assitance by teachers in small groups in discussing question and practising exercises which containing HOTS. This resonates with previous study, stated that the role of teachers in virtual classrooms and face-to-face classes when giving instructions is substantial aspect as a vehicle to make students actively involved in teaching and learning activities to promote critical thinking of students (Mandernach, Babutzke, \& Manker , 2009)

The exercises containing HOTS was deliberately given by the teacher. It included the question of the previous UNBK questions in the virtual classroom. In the short term period, this is intended to prepare the 12th grade students of MIA in facing the upcoming national exam in 2019. 
Flipped classroom using WhatsApp especially virtual classroom of Rumah Belajar is helpful for the teacher in promoting HOTS for students. The 12 MIA students of SMA N 5 Kupang became more critical, independent, and active in using HOTS which then hoped to be able to solve problems in the future. The combined use of WhatsApp and Rumah Belajar shed the light to a greater opportunity for promoting students' Higher Order Thinking Skills.

\section{CONCLUSION}

Flipped classroom both using WhatsApp and virtual classroom of Rumah Belajar has a positive impact on the maths learning activities for 12 MIA students of SMA N 5 Kupang NTT. The majority of focus groups favour the combined use of WhatsApp and virtual classroom of Rumah Belajar simultaneously in flipped classrooms because these two learning platforms complement each other. The gap found in the use of WhatsApp are filled by the advantages in the virtual classroom of Rumah Belajar.

Apart from a few technical problems in Rumah Belajar (see table 1), students mentioned that they benefited from features in the virtual classroom of Rumah Belajar such as exercises, assignments and online examinations. The 12 MIA students admitted that they were better prepared to face the upcoming UNBK (Computer-Based National Examination).

The amount of time used in face- to-face classroom is more efficient to spend more for practising UNBK in small groups. This is very beneficial for students because the questions given to practise both in virtual classroom and face-to-face classroom are questions that containing HOTS.

In another word, flipped classroom by using virtual classroom of Rumah Belajar has a positive impact on $12 \mathrm{MIA}$ students in promoting their HOTS so that in the short term they are better prepared to face the UNBK next year. Furthermore, the students also stated that they became more well prepared, more independent, more active. This led them to be critical thinkers. For the long term benefit, it can be said that they learnt to have the ability to think critically in dealing with problems which is considered critical in the education 4.0. The last but not the least, this research is a pure qualitative study. In the future, quantitative or mixed qualitative and quantitative research is needed to confirm the results of this current research in investigating flipped classroom using virtual classroom of Rumah Belajar to promote higher order thinking skills (HOTS) of students in learning mathematics and other subjec

\section{Appendix 1}

List of Questions in Focus Group Discussion:

1) How do you perceive the math flipped classroom using WhatsApp? (Efficient or not)

2) What are the advantages of using WhatsApp as an online learning platform? Please explain it.

3) What are the disadvantages of using WhatsApp as an online learning platform? Please explain it.

4) How do you perceive the math flipped classroom using virtual classroom of Rumah Belajar? (Efficient or not)

5) What are the advantages of using virtual classroom of Rumah Belajar as an online learning platform? Please explain it. 
6) What are the disadvantages of using virtual classroom of Rumah Belajar as an online learning platform? Please explain it.

7) For the upcoming learning experiences, which one do you prefer among the following three choices? (Elaborate your reasons)

a. Flipped Classroom: only use WhatsApp without virtual classroom of Rumah Belajar

b. Flipped Classroom: only use virtual classroom of Rumah Belajar without WhatsApp.

c. Flipped Classroom: Use both, WhatsApp and virtual classroom of Rumah Belajar.

8) How about your experiences in working on exercises, assignments and online test which containing higher order 
thinking skills in our flipped math classroom using WhatsApp and Rumah Belajar?

\section{References}

Amresh, A., Carberry, A. R., \& Femiani, J. (2013). Evaluating the Effectiveness of Flipped Classrooms for Teaching CS1. Paper Presented at the Frontiers in Education Conference, Oklahoma City, 733-735.

Falode, O. C., Gambari, A. I., Shittu, T., Gimba, R. W., Falode, M. E., \& Awoyemi, D. I. (2015). Effectiveness Of Virtual Classroom In Teaching And Learning Of Senior Secondary School Mathematics Concepts In Minna, Nigeria. oobi:Indo-African Journal of Educational Research, 3(4), 1-4.

Fulton, K. (2012). Upside Down and Inside Out: Flip Your Classroom to Improve Student Learning. ISTE (International Society for Technology in Education), 4(1), 12-16.

Griesemer, J. A. (2014). using-social-media-to-enhance-students-learning-experiences.

Kustijono, R., \& Zuhri, F. (2018). The use of Facebook and WhatsApp application in learning process of physics to train students' critical thinking skills. IOP Conf. Ser.: Mater. Sci. Eng.

Lenhart, A., Purcell, K., Smith, A., \& Zickuhr, K. (2010). Social Media \& Mobile Internet Use among Teens and Young Adults. Millennials. Pew Internet \& American Life Project.

Liu, Y. (2010). Social Media Tools as a Learning Resource. Journal of Educational Technology Development and Exchange, 3, 101-114.

Livingstone, S. (2012). Critical reflections on the benefits of ICT in education. Oxford Review of Education, 38(1), 9-24.

Mandernach, J., Babutzke, J. L., \& Manker, L. R. (2009). :öjiThe Role of Instructor Interactivity in Promoting Critical Thinking in Online and Face -to-Face Classrooms. MERLOT Journal of Online Learning and Teaching, 5 (1).

Martin, F., \& Parker, M. A. (2014). Use of Synchronous Virtual Classrooms: Why, Who, and How? MERLOT Journal of Online Learning and Teaching, 10(2), 192-210.

Sayan, H. (2016). Affecting Higher Students Learning Activity By Using Whatsapp. European Journal of Research and Reflection in Educational Sciences, 4(3).

Strayer, J. F. (2012). How Learning in an Inverted Classroom Influences Cooperation, Innovation and Task Orientation. Learning Environments Research. 15, 171-193.

Williams, A., \& Katz, L. (2001). The Use of Focus Group Methodology in Education: Some Theoretical and Practical Considerations. . International Electronic Journal for Leadership in Learning, 5. 